\title{
A High-Resolution Study of the OH-Stretch Fundamental of Methanol ${ }^{1}$
}

\author{
R. Gary Lee, Robert H. Hunt, and Earle K. Plyler \\ Department of Physics, The Florida State University, Tallahassee, Florida 32306 \\ AND \\ David M. Dennison \\ Department of Physics, The Universily of Michigan, Ann Arbor, Michigan 48104
}

\begin{abstract}
The OH-stretch fundamental of $\mathrm{CH}_{3} \mathrm{OH}$ has been observed with $0.025 \mathrm{~cm}^{-1}$ resolution between 3430 and $3940 \mathrm{~cm}^{-1}$ and the resulting spectrum deconvoluted using the procedure of $P$. A. Jansson. Approximately 600 lines have been assigned to a total of $67 P$-or $R$-branch series and some 30 excited state levels have been detcrmined. Of thesc, 14 belong to the lowest torsional state with $n=0,13$ to $n=1$ and 3 to $n=2$. A nonlinear least-squares fit to these levels varying the major parameters used by Y. Y. Kwan and D. M. Dennison in their analysis of the normal state produces an rms deviation between observed and calculated levels of $0.51 \mathrm{~cm}^{-1}$. Variation of all the parameters including those of the smaller Kirtman perturbation terms produces only a slight improvement in the fit. Both analyses yield a barrier height of $411 \mathrm{~cm}^{-1}$ in the excited vibrational state as compared to the normal state value of $373 \mathrm{~cm}^{-1}$. A number of unexplained anomalies appear in the spectra including large and irregular changes in the coefficient of $J^{2}+J$ for different torsion-rotation states.
\end{abstract}

\section{INTRODUCTION}

The methyl alcohol molecule possesses an intense and complex rotation-vibration spectrum which extends almost continuously from the microwave region to the limit of the visible spectrum. The longer wavelength portion up to approximately 11 microns arises from transitions between rotational states including hindered rotation or torsion. This part of the spectrum has been the subject of a number of investigations $(1-3)$ in recent years which have led to a determination of the molecular constants of methanol as well as to accurate methods for calculating the torsion-rutation levels of the normal or non-vibrating molecule. The shorter wavelength portion of the infrared methanol spectrum results from vibrational transitions which are coupled with rotation to produce bands with a complex and often overlapping fine structure.

The methanol spectrum and its interpretation are particularly interesting for a number of reasons. In the first place the size of the barrier to rotation of the hydroxyl group relative to the methyl group, namely $373 \mathrm{~cm}^{-1}$, is of the same order of magnitude as thermal energy, $k T=204 \mathrm{~cm}^{-1}$, at room temperature. Thus, spectroscopic transitions are observed between levels some of which lie well below the top of the barrier, some in the neighborhood of the barrier and some well above it. This means that the barrier

${ }^{1}$ Work supported in part under NASA grant NGR 10-004 056. 
height as well as many of the other molecular constants can be determined with considerable precision.

The occurrence and origin of the barrier is of interest since it depends upon the interaction of forces between distant parts of the molecule, namely the hydroxyl group and the methyl group with its three equivalent hydrogens. A simple dimensional argument leads to the conclusion that the barrier height should be a sensitive, nonlinear, function of the distance of the hydroxyl hydrogen from the plane of the methyl hydrogens as well as from the axis of the methyl group. It should therefore depend upon the vibrational state of the molecule. This expectation is substantiated by a number of pieces of experimental evidence. The analysis of the normal state rotation-torsion lcvels of $\mathrm{CH}_{3} \mathrm{OH}$ and of $\mathrm{CD}_{3} \mathrm{OD}$ by Kwan and Dennison (3) showed that the barrier height for fully deuterated methanol is lower by about $11 \mathrm{~cm}^{-1}$ than for the undeuterated molecule. This can be attributed to the smaller amplitude of the zero-point vibrations in the deuterated species. The vibrational band of $\mathrm{CH}_{3} \mathrm{OH}$ centered at $1034 \mathrm{~cm}^{-1}$ has been observed by Woods and Peters (2). This band may be described to good approximation as a parallel vibration due to the $\mathrm{C}-\mathrm{O}$ stretch. Their resolution of about $0.07 \mathrm{~cm}^{-1}$ was sufficient to show clearly that the relatively simple structure of a parallel band was altered due to the fact that the effective barrier height had been changed by the vibration. They found that the barrier for the excited state was $19 \pm 2 \mathrm{~cm}^{-1}$ higher than for the normal state. Lees (4), from microwave measurements, corroborated this result and obtained a figure of about $22 \mathrm{~cm}^{-1}$. Lees (5) has also found other "hot band" lines in the microwave region for which the excited state barrier height is approximately $50 \%$ higher than for the normal state, namely, $557 \pm 35 \mathrm{~cm}^{-1}$. Lees suggests that this vibration is one which involves a rocking of the methyl group relative to the hydroxyl and notes that this motion might be expected to have a large effect on the effective barrier height.

The first portion of the present investigation consists of an experimental measurement of the absorption spectrum of $\mathrm{CH}_{3} \mathrm{OH}$ from 3430 to $3940 \mathrm{~cm}^{-1}$. This region encompasses only one fundamental band, namely, the $\mathrm{OH}$ stretching vibration which is centered around $3680 \mathrm{~cm}^{-1}$. The resolution attained (before deconvolution) averaged $0.025 \mathrm{~cm}^{-1}$. This band consists of superimposed perpendicular and parallel fine structure lines, but from the geometry of the molecule and the rough assumption that the electric moment vibrates along the equilibrium $\mathrm{OH}$ line, one would estimate that the intensities of the perpendicular and parallel components of the total band would be in the ratio of about 6 to 1 . Because of this expected difference in intensity, attention will be centered on the perpendicular component lines.

In the second part of the present work we have succeeded in identifying about 600 of the experimentally measured lines. From these, through various combination relations, the energy levels of the vibrating molecule can be determined and hence many of the molecular constants, including the effective barrier height, can be calculated. Our analysis closely follows that employed by Kwan and Dennison (3) and will use their notation. A brief description of the general structure of the energy levels and the resulting spectrum may be helpful. The details are given in Ref. (3).

In the normal state where all vibrational quantum numbers (with the exception of the torsion) are equal to zero, the levels are described by four numbers. The first of these, $n$, might be said to label the state of torsional vibration, although this designation 
is only strictly correct for molecules with high barriers. The second number, $\tau$, can take on the three values 1,2 , or 3 and relates to the threefold nature of the potential minima and the tunneling through the three equivalent potential barriers. The third number, $J$, measures the total angular momentum of the system while $K$ represents (to a very high approximation) the component of $J$ along an axis which may be denoted as the symmetry axis of the molecule. This designation is meaningful since the methanol molecule is very nearly a symmetrical rotator with two of the principal moments of inertia very nearly equal while the third is much smaller.

The energy levels are best discussed, and also calculated, by beginning with zerothorder terms which describe an axially symmetrical model consisting of two rigid parts (representing the hydroxyl and methyl groups) which can rotate with respect to each other along the symmetry axis subject to a hindering potential with three equal minima. It is assumed that the hindering potential has the sinusoidal form $\frac{1}{2} V_{3}(1-\cos 3 \gamma)$. These zeroth-order terms may be divided into two parts of which one is the usual rotational energy for a symmetrical top,

$$
\left(E_{R}^{0}\right) / h c=\frac{1}{2}(B+C)\left(J^{2}+J\right)+\left[A-\frac{1}{2}(B+C)\right] K^{2} .
$$

$A, B$, and $C$ are functions of the inertial constants [see Ref. (3)] but to good approximation $A$ is proportional to the inverse of the small moment of inertia while $B$ and $C$ are, respectively, proportional to the inverse of the large moments of inertia.

The energy contribution due to the mutual rotation of the two parts of the model is denoted by $E_{n \tau} K^{0}$ and, as indicated, is a function of the quantum numbers $n, \tau$, and $K$. The molecular constants which determine $E_{n \tau K}{ }^{0}$ are the barrier height, $V_{3}$, and $F$. $F$ is a function of moments and products of inertia but, again to a good approximation, it is proportional to $\left(I a_{1}+I a_{2}\right) /\left(I a_{1} \cdot I a_{2}\right)$ where $I a_{1}$ and $I a_{2}$ are, respectively, the moments of inertia of the hydroxyl and methyl groups about the symmetry axis of the model. The wavefunctions for the zeroth-order model may be calculated and these serve as the basis functions for evaluating the energy contributions from perturbation terms.

The differences betwecn a model which morc realistically represents the methanol molecule and the zeroth-order model described above are accounted for by perturbation terms. These include terms due to asymmetry, to the deviation of the potential energy from sinusoidal form, and to the nonrigidity of the molecule. The latter include the usual certrifugal distortion terms with the constants $D_{J J}, D_{J K}, D_{K K}$ as well as the Kirtman terms (6) which contain the Kirtman semiempirical constants $F_{V}, G_{V}, L_{V}$, $k_{1}, \ldots, k_{7}$.

The total contribution from the above perturbation terms is relatively small when compared with the energies of the zeroth-order model and this fact will become important in analyzing the fine structure of the $\mathrm{OH}$ vibration band. Thus, for $n=0$ and for the typical range of $K=0$ to 10 the average contribution of the perturbations to the normal state energies is $0.42 \mathrm{~cm}^{-1}$ with a maximum value of $0.65 \mathrm{~cm}^{-1}$. The corresponding range of the zeroth-order energies is from $128 \mathrm{~cm}^{-1}$ to $480 \mathrm{~cm}^{-1}$. For $n=1$ the figures are: average perturbation $0.63 \mathrm{~cm}^{-1}$ and maximum $1.67 \mathrm{~cm}^{-1}$ over a range 3.30 to $760 \mathrm{~cm}^{-1}$, while for $n=2$ the corresponding numbers are $2.69,8.68,480$, and 1000 $\mathrm{cm}^{-1}$. 
The appearance of the fine structure of the $\mathrm{OH}$ stretching band, as noted, will be dominated by lines from the perpendicular component for which the selection rule is $\Delta K= \pm 1$. The total fine structure of a perpendicular band may be described as consisting of the superposition of many subbands each with its own $P, Q$, and $R$ branches. The positions of the lines in any of these branches is given as the sum of what is defined as a $Q$-branch origin and a power series in $J$. The $Q$-branch origin in the present case is the sum of a large term, $\nu_{0}$, the fundamental energy of vibration, and the difference, $\left(n^{\prime} \tau^{\prime} K^{\prime}\right)^{*}-(n \tau K)$, between the hypothetical $J=0$ energy levels of the appropriate excited and normal torsion-rotation states. The spacing of the lines in the $P$ and $R$ branches will be approximately equal to $(B+C)$ or $1.61 \mathrm{~cm}^{-1}$ but will be modified by the Kirtman perturbation terms as well as by the fact that $\left(B^{*}+C^{*}\right)$ for the excited state will differ slightly from $B+C$ for the normal state. The analysis of this fine structure is complicated by three factors. First, at room temperature there is an appreciable fraction of molecules in torsional states with $n>0$. This, in conjunction with the selection rules, $\Delta n$ arbitrary, $\Delta K= \pm 1$ and $\tau=1 \leftrightarrow 2,2 \leftrightarrow 3$, or $1 \leftrightarrow 3$, results in a very large number of subbands of appreciable intensity in the region of observation. Second, the oscillatory behavior of the internal levels as a function of $K$ leads to a very irregular spacing of the $Q$-branch origins. Third, the subband intensities do not follow a rigid symmetric top pattern as the internal-rotation intensity factors are also periodic functions of $K(7)$.

Fortunately, the energy levels of the normal state are known with considerable precision (3). As will be discussed in the analysis, the use of combination differences involving these levels proved to be the indispensable tool in making identifications. In all, $67 P$ - and $R$-branch series have been identified and these have resulted in the determination of 30 excited state levels of which 14 belong to the lowest torsion state with $n=0$, while 13 have $n=1$ and 3 have $n=2$. An analysis based upon the methods used in the previous investigations $(1-3)$ of the normal or nonvibrating molecule serves to explain the principal features of the observed spectrum and shows that the barrier height for the OH-stretch excited state is approximately $10 \%$ higher than its value for the normal state. However, the agreement between the observed levels and those calculated using the molecular constants from a least-squares fit to the excited state is definitely poorer than the corresponding agreement for the normal state levels. It appears probable that these deviations may well result from resonances with certain overtone or combination levels which fall in the region of the $\mathrm{OH}$ fundamental.

\section{EXPERIMENTAL DETAILS}

The spectra were recorded with a $3 \mathrm{~m}$ focal-length, four-pass vacuum spectrometer (8) equipped with a $25 \mathrm{~cm}, 300$ ruling-per-mm grating blazed at $3 \mu \mathrm{m}$ in second order. The source was a $650 \mathrm{~W}$ quartz-iodine lamp operated in a helium flushed housing with the remainder of the optical path under vacuum. Methanol vapor was introduced directly into the spectrometer vacuum chamber which provided an optical path of $34.2 \mathrm{~m}$. Pressures ranged from 0.03 Torr at the band center to 0.85 Torr in the wings.

Two sets of scans were made. The first consisted of a low-noise, digitally recorded spectrum (signal-to-noise ratio of better than 200 to 1) which was subsequently deconvoluted by the method of Jansson et al. ( $(9)$. The time constant for these scans was 5 


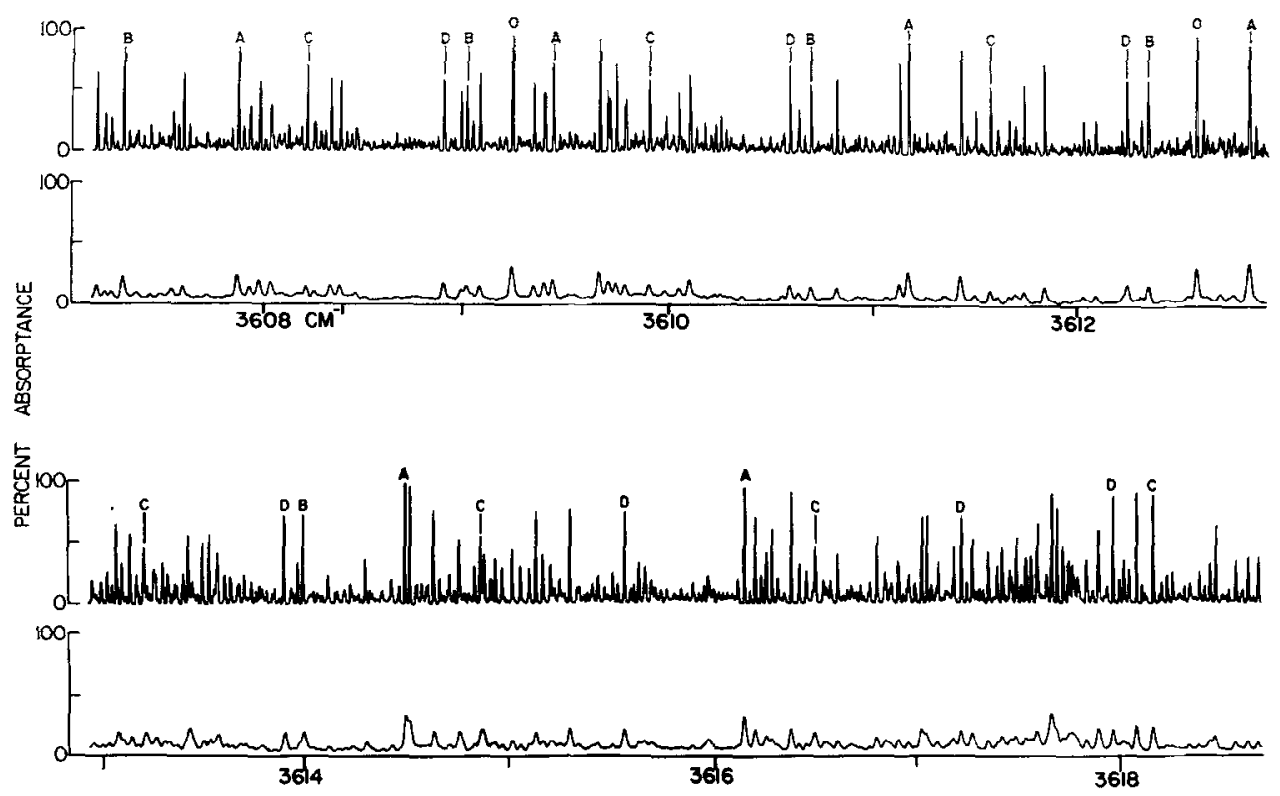

FIG. 1. Observed and deconvoluted spectra on the $P$-branch side of the $\mathrm{OH}$-stretching band of $\mathrm{CH}_{3} \mathrm{OH}$ showing some of the identified series in this region. Lines labeled $A, B, C$ are members of the series $(037 J-1)^{*} \leftarrow(028 J),(018 J-1)^{*} \leftarrow(039 J)$, and $(017 J-1)^{*} \leftarrow(038 J)$, respectively. The lines labeled $D$ are part of a series as yet not identified. Strong water lines are indicated by circles.

sec and the instrument response function width at half-maximum varied from 0.020 to $0.028 \mathrm{~cm}^{-1}$. The number of data points recorded over this width varied from 10 to 20 .

The second set of scans was for the purpose of calibration and was made at similar resolution but with the time constant reduced to $1 \mathrm{sec}$. Between 3560 and $3750 \mathrm{~cm}^{-1}$ $\mathrm{CO}_{2}$ was mixed with the methanol vapor and the calculated line positions given by Gordon and McCubbin (10) for the $10^{\circ} 1-000$ and $02^{\circ} 1-000$ bands were used as calibration standards. For the remaining portion of the spectrum, which was obtained somewhat later, the standards were thorium emission lines $(10,11)$. These lines were interspersed throughout the methanol spectrum with the aid of two mirrors mounted on rotary solenoids. Switching of the solenoids caused the infrared illumination of the entrance slit to be replaced with the emission from the thorium lamp while simultaneously diverting the output of the exit slit from the $\mathrm{PbS}$ detector to the photomultiplier.

The frequencies of unblended methanol lines were measured from the calibration spectra to an estimated accuracy of $\pm 0.005 \mathrm{~cm}^{-1}$ and the results used as secondary standards to determine the remaining line positions in the deconvoluted spectrum. Figures 1-3 show three regions of the spectrum as observed and after deconvolution. Several series of lines, identified using the methods discussed in the analysis are indicated.

In obtaining the deconvoluted spectrum, two initial 15 point cubic polynomial smooths of the raw data were performed and the result used as the observed spectrum. A total of fifteen iterations using a Gaussian approximation to the response function 

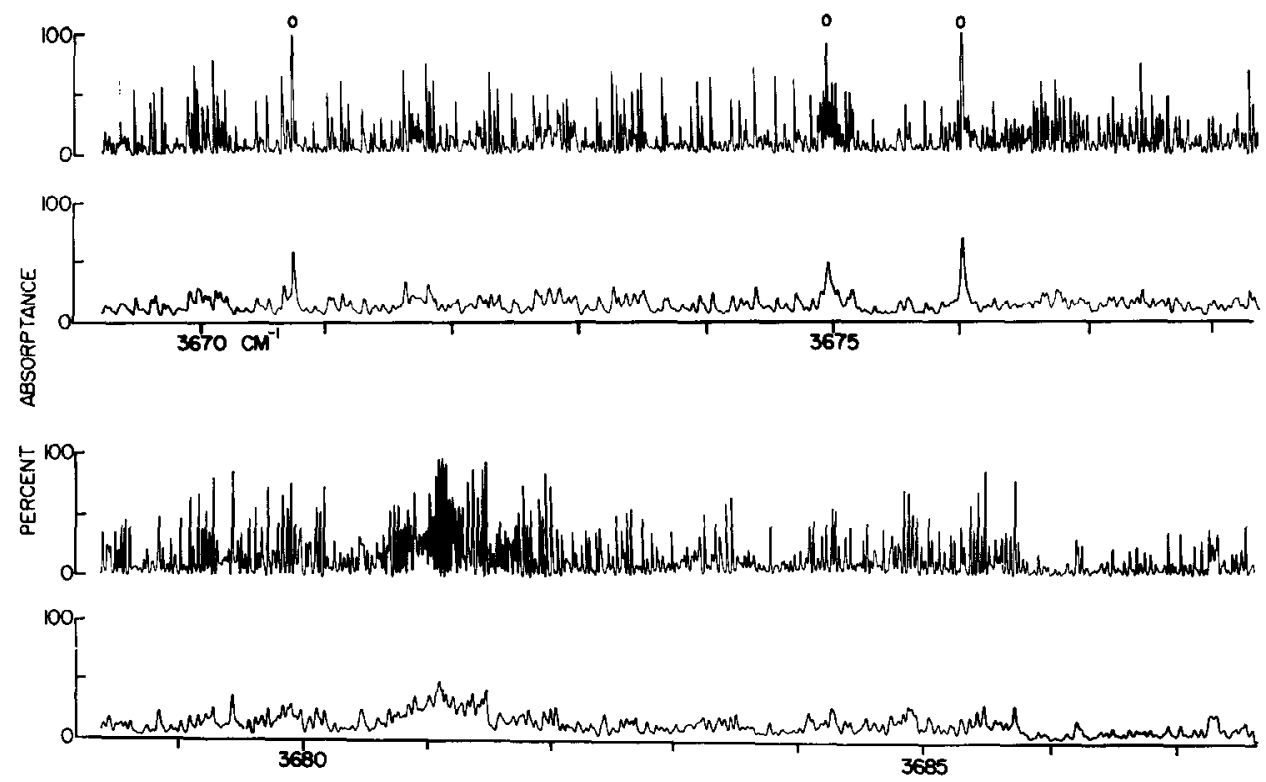

Fig. 2. Observed and deconvoluted spectra in the region of the band center. The pressure was approximatcly 30 microns with a path of $34 \mathrm{~m}$. Strong water lines are indicated by circles.
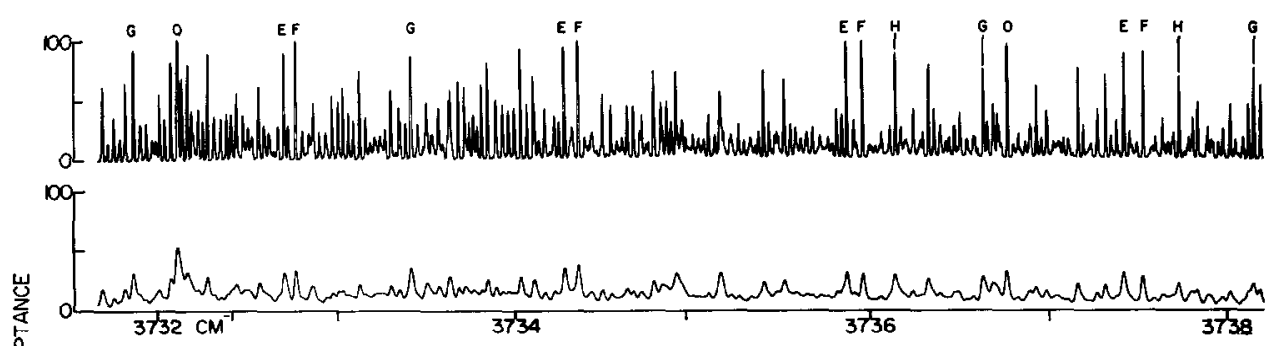

告
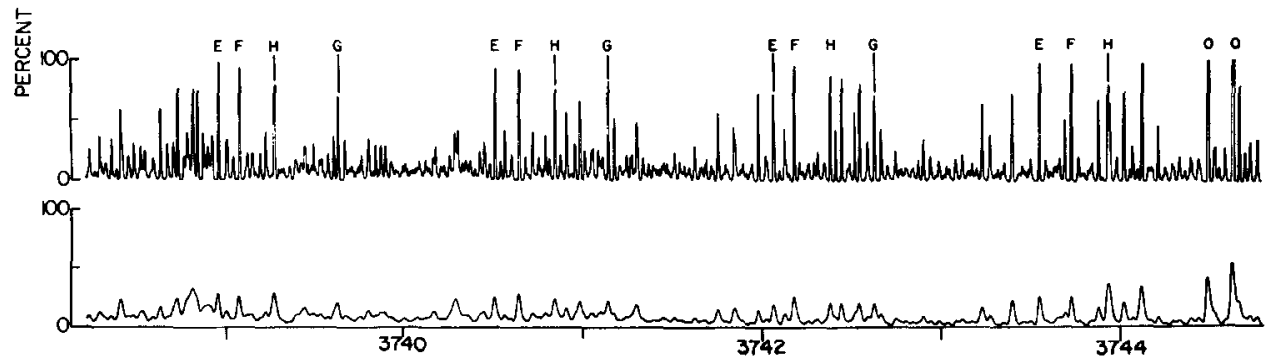

Fig. 3. A section of the $R$-branch side of the $\mathrm{OH}$-stretch fundamental showing observed and deconvoluted spectra as well as some of the lines identified in this region. Lines labeled $E, F, G, H$ are members of the series $(025 J+1)^{*} \leftarrow(034 J),(016 J+1)^{*} \leftarrow(025 J),(037 J+1)^{*} \leftarrow(016 J)$, and $(026 J+1)^{*} \leftarrow$ $(035 J)$. Strong water lines are indicated by circles. 
were made with 15 point cubic smoothing of the results of the first five iterations [see Ref. (9)].

In all, the frequencies of 8900 lines were determined. These do not include lines of $15 \%$ absorptance or less in the deconvoluted spectrum inasmuch as such lines are very numerous and somewhat uncertain because of the noise level present and some uncertainty in placing the level of zero absorptance.

\section{ANALYSIS}

Since the spectrum exhibited only a few obvious $Q$ branches, the initial step in the analysis was to search for series of lines having the expected $P$ - and $R$-branch spacing of about $1.6 \mathrm{~cm}^{-1}$. Originally such series were obtained directly from the traces; later the more efficient method of making Loomis-Wood plots was used. The next step was to determine which transitions $\left(n^{\prime} \tau^{\prime} K^{\prime} J^{\prime}\right)^{*} \leftarrow(n \tau K J)$ were likely to occur in the region of a measured series. For performing this step two important aids were available. The first and most important was a table giving the positions and intensities of $P_{-}, Q_{-}$, and $R$-branch lines for transitions between the normal state levels, $\left(n^{\prime} \tau^{\prime} K^{\prime}\right) \leftarrow(n \tau K)$. We wish to express our gratitude to Dr. Y. Y. Kwan for calculating this table and making it available to us. The second was a qualitative estimate of the differences to be expected between normal and excited state levels which possess the same values of the quantum numbers $n, \tau, K, J$. The largest contribution to such differences is, of course, the $\mathrm{OH}$ vibration frequency which from inspection of the spectrum must have a value of approximately $3680 \mathrm{~cm}^{-1}$. The next-largest contributions should come from the zerothordcr rotation terms, $E_{R}{ }^{0}$ and $E_{n \tau K^{0}}$, as a result of small changes in the barrier height and the effective moments of inertia. From our present knowledge, it is not possible to make a meaningful estimate of the change of barrier height, although, considering the nature of the $\mathrm{OH}$ vibration, one might guess that it would be larger than the change associated with $\mathrm{CO}$ vibration but not as large as that for the rocking vibration. Both $E_{R}{ }^{0}$ and $E_{n \tau}{ }^{0}$ are influenced by changes in the effective moments of inertia. If the $\mathrm{OH}$ stretch fundamental is approximated by a vibration of the hydrogen relative to the oxygen in the hydroxyl group and if the potential energy for such a displacement is taken equal to that for the high-frequency vibration of $\mathrm{H}_{2} \mathrm{O}$ (including the anharmonic terms), it is quite easy to calculate the effective moments of inertia for the excited state. The results of such a calculation are that the coefficient of $\left(J^{2}+J\right)$ of $E_{R}{ }^{0}$, namely, $(B+C) / 2$, should decrease by about $0.0015 \mathrm{~cm}^{-1}$, the effective value of $I a_{1}$ should increase by about $6 \%$ while $I a_{2}$ should be virtually unchanged. Of course calculations of this sort should only be used as a guide in making the analysis of the observed spectrum. Considered in this way they indicate that the zeroth-order rotation terms for the excited state may be expected to differ from the corresponding terms of the normal state by a few waves per centimeter for $n=0$ states and progressively more as $n$ increases or, in other words, by a few percent of the normal state values.

Since the perturbation terms, which contain the centrifugal and Kirtman constants, are so much smaller than the zeroth-order part of the energy in the normal state, it seems reasonable to expect that changes in them would be of lesser importance in the analysis.

With the above as guidelines as to the region of the spectrum to be searched for the series arising from the transition $\left(n^{\prime} \tau^{\prime} K^{\prime} J^{\prime}\right)^{*} \leftarrow(n \tau K J)$, it was possible to limit the 
assignments of the stronger more obvious series to a fairly small number of possibilities. The final step was to look for a companion scries belonging to the transition $\left(n^{\prime} \tau^{\prime} K^{\prime} J^{\prime}\right)^{*_{\leftarrow}}$ $\left(n^{\prime \prime} \tau^{\prime \prime} K^{\prime \prime} J^{\prime \prime}\right)$. When such a candidate series was found, combination differences, $\left(n^{\prime \prime} \tau^{\prime \prime} K^{\prime \prime} J^{\prime \prime}\right)-(n \tau K J)$, which involve only normal state levels, were formed and compared to those calculated from the normal state levels. Since the latter are accurately known (to the order of several thousandths of $\mathrm{c} \mathrm{cm}^{-1}$ for $n=n^{\prime \prime}=0$ ), good agreement between observed and calculated differences furnished strong corroboration for the correctness of the identifications.

As an example, consider the series of $R$-branch lines shown in the first column of Table I. The first line of the series lies at $3729.601 \mathrm{~cm}^{-1}$. This frequency is approximately $50 \mathrm{~cm}^{-1}$ higher than the apparent band center at about $3680 \mathrm{~cm}^{-1}$. A search of the table of normal state frequencies and intensities calculated by $\mathrm{Kwan}$ shows that an intense series of $R$-branch lines originating from the transitions $(016 J) \leftarrow(025 J-1)$ should start at $48.5 \mathrm{~cm}^{-1}$. Assuming that the observed series in question is indeed $(016 J)^{*} \leftarrow$ $(025 J-1)$, one can now look for the $P$-branch series $(016 J)^{*} \leftarrow(037 J+1)$, which according to the calculations should also be intense. This series is readily found and the observed lines are listed in the second column of Table I. The third column gives the observed differences, $(037 J+1)-(025 J-1)$, between corresponding lines of the $R$ and $P$ branches, whereas column 4 gives these differences as calculated from the normal state energy levels $(1,3)$. The differences, $\delta$, between the observed and calculated values appear in the last column. These latter differences are small, of the order of the experimental accuracy, and appear to be randomly distributed. It seems reasonable to conclude that (1) the two series $(016 J)^{*} r \cdot(025 J-1)$ and $(016 J)^{*} \leftarrow(037 J+1)$ have been correctly identified, (2) the normal state energy levels $(025 J)$ and $(037 J)$ are indeed known with an accuracy at least as high as several thousandths of a wave per centimeter, and (3) the accuracy of the present observations do not lie outside this same range.

\begin{tabular}{|c|c|c|c|c|c|}
\hline $\mathrm{J}$ & $\left(R_{J-1}\right)_{\text {obs }}$ & $\left(P_{\mathrm{J}+1}\right)^{\text {obs }}$ & $\left(R_{J-1}-P_{J+1}\right)^{\text {obs }}$ & $\left(R_{J-1}-P_{J+1}\right)_{\text {calc }}$ & $\delta$ \\
\hline 6 & 3729.601 & 3634.416 & 95.185 & 95.184 & +0.001 \\
\hline 7 & 3731.191 & 3632.794 & 98.397 & 98.403 & -0.006 \\
\hline 8 & 3732.779 & 3631.156 & 101.623 & 101.621 & +0.002 \\
\hline 9 & 3734.352 & 3629.517 & 104.835 & 104.838 & -0.003 \\
\hline 10 & 3735.942 & 3627.881 & 108.061 & 108.053 & +0.008 \\
\hline 11 & 3737.509 & 3626.251 & 111.258 & 111.266 & -0.008 \\
\hline 12 & 3739.076 & 3624.594 & 114.482 & 114.478 & +0.004 \\
\hline 13 & 3740.643 & 3622.951 & 117.692 & 117.687 & +0.005 \\
\hline 14 & 3742.194 & 3621.309 & 120.885 & 120.895 & -0.010 \\
\hline
\end{tabular}




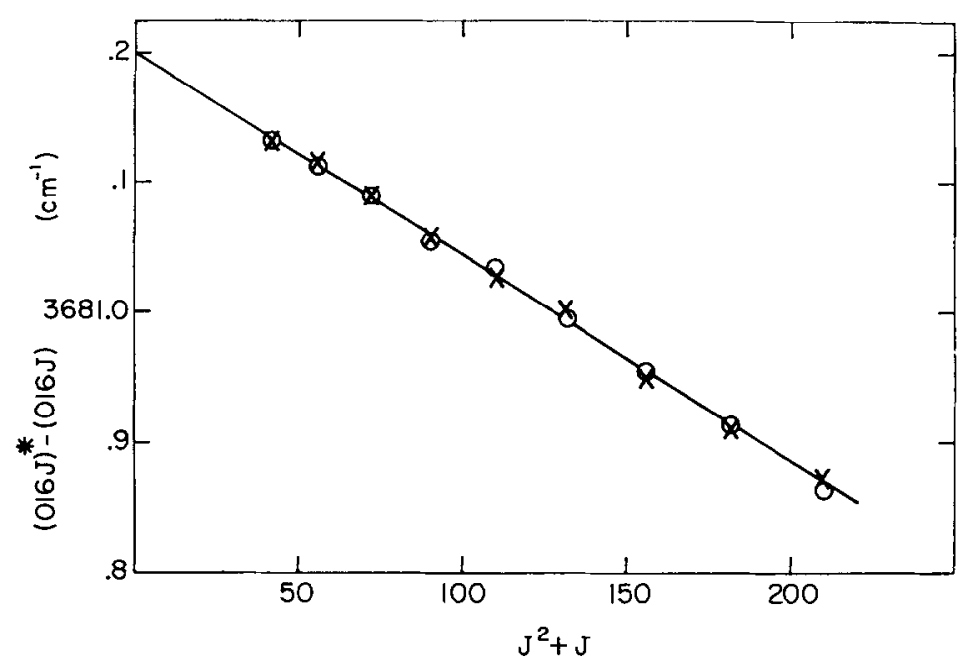

Fig. 4. Plots of $(016 J)^{*}-(016 J)$ versus $J^{2}+J$. The circles indicate points obtained from $\left[(016 J)^{*}\right.$ $-(025 J-1)]_{\mathrm{obs}}+[(025 J-1)-(016 . J)]_{\mathrm{ca} I_{0}}$ whereas the crosses are from $\left[(016 J)^{*}-(037 J+1)\right]_{\text {obo }}$ $+[(037 J+1)-(016 J)]_{\text {ealc. }}$.

These data can be plotted in a way which shows the consistency in the assignment of the two series and at the same time determines two important pieces of information regarding the excited state levels. Suppose that to each observed line of the $R$ branch one adds the normal state quantity, $(025 J-1)-(016 J)$, and to each $P$-branch line, $(037 J+1)-(016 J)$. The results, namely, $(016 J)^{*}-(016 J)$, which should be the same for each pair of lines, will, however, differ by the amounts which appear in the last column of Table I. Now the differences, $(016 J)^{*}-(016 J)$, as discussed earlier, will contain as the largest term the fundamental $\mathrm{OH}$ vibration frequency. Next they will possess a term of the order of perhaps a few waves per centimeter or less which arises from the difference in the effective molecular constants such as $I a_{1}, I a_{2}$, and $V_{3}$ in the excited state. This term is a function of the quantum numbers $n, \tau$, and $K$ but not of $J$. Finally, $(016 J)^{*} \leftarrow(016 J)$ may be expected to depend upon a polynomial in $\left(J^{2}+J\right)$. The first term, which is proportional to $\left(J^{2}+J\right)$, should be dominant and, accordingly, $(016 J)^{*}-(016 J)^{2}$ has been plotted against $\left(J^{2}+J\right)$ as shown in Fig. 4. Clearly the points'obtained from both the $P$ - and $R$-branch series are well represented by a single straight line which in this case has the equation $(016 J)^{*}-(016 J)=$ $3681.198-0.0016\left(J^{2}+J\right) \mathrm{cm}^{-1}$. The intercept, denoted by $(016)^{*}-(016)$, measures the $J$ independent part of the change in energy brought about by the $\mathrm{OH}$ vibration. The slope of the straight line in Fig. 4 represents the major effect of the vibration on the $J$ dependent part of the energy. As mentioned earlier, the largest contribution to the effect should be the term $\frac{1}{2}\left[(B+C)^{*}-(B+C)\left(J^{2}+J\right)\right.$. Our estimate of the coefficient of $\left(J^{2}+J\right)$, namely, $-0.0015 \mathrm{~cm}^{-1}$, is in excellent agreement with the slope shown in Fig. 4. Unfortunately, it turns out that this agreement is somewhat fortuitous.

All the series that have been identified, some 67 in number, have been treated in the above way and plots similar to Fig. 4 have been constructed. The intercepts and slopes so obtained are listed in Table II together with the series from which they were derived. 
In one instance, namely the transition $(110)^{*} \leftarrow(231)$, both $P$ - and $R$-branch series were observed and combination differences involving only the lower state, (231), served for the identification. For the most part the points were well represented by straight lines although in some cases an increasing curvature for the larger values of $J$ was apparent thus indicating the existence of a significant term proportional to $\left(J^{2}+J\right)^{2}$.

A few series exhibited what appears to be the effect of a local resonance at intermediate $J$ values and did not give smooth plots. Two pairs of such series $(234)^{*} \leftarrow(225)$, $(234)^{*} \leftarrow(113)$ and $(037)^{*} \leftarrow(016),(037)^{*} \leftarrow(028)$ have been identified. Figure 5 shows the plots obtained from the latter pair. The slope and intercept listed in Table II were obtained from the dashed line which is drawn so as to connect the end points on the assumption that these arise from relatively unperturbed lines.

Where two series have been found each of which starts from a normal state having the torsional number $n=0$, the usual situation was of the type shown in Fig. 4 in which

Table II. Results from the plots of $(n \tau K J) *-(n \tau K J)$.

\begin{tabular}{|c|c|c|c|}
\hline Intercept & $\left(\mathrm{cm}^{-1}\right)$ & slope & Series Employed \\
\hline$(016)^{*}-(016)$ & $=3681.198$ & -0.0016 & $(016)^{7}-(025),-(037),-(125)$ \\
\hline$(017)^{*}-(017)$ & 80.290 & -0.0019 & $(017)^{*}-(026),-(038)$ \\
\hline$(018)^{*}-(018)$ & 79.923 & -0.0026 & $(018)^{*}-(027),-(039)$ \\
\hline$(019)^{*}-(019)$ & 79.539 & -0.0028 & $(019)^{\star}-(028),-(0310)$ \\
\hline$(024)^{*}-(024)$ & 81.142 & -0.0022 & $(024)^{*}-(033),-(015),-(115)$ \\
\hline$(025)^{*}-(025)$ & 81.556 & -0.0023 & $(025)^{*}-(034),-(016),-(116),-(134$ \\
\hline$(026)^{*}-(026)$ & 81.400 & -0.0024 & $(026)^{*}-(035),-(017),-(117)$ \\
\hline$(027)^{*}-(027)$ & 81.964 & -0.0024 & $(027)^{*}-(036),-(018)$ \\
\hline$(028)^{*}-(028)$ & 81.406 & -0.0028 & $(028)^{*}-(037),-(019)$ \\
\hline$(0211)^{\star}-(0211)$ & 80.565 & -0.0028 & $(0211)^{*}-(0310),-(0112)$ \\
\hline$(034)^{*}-(034)$ & 85.550 & -0.0050 & $(034)^{*}-\left(\begin{array}{ll}01 & 3\end{array}\right),-\left(\begin{array}{ll}02 & 5\end{array}\right),-(125)$ \\
\hline$(037)^{\star}-\left(\begin{array}{ll}03 & 7\end{array}\right)$ & 82.190 & -0.0026 & $(037)^{\star}-(016),-(028)$ \\
\hline$(0310)^{\star}-(0310)$ & 80.104 & -0.0015 & $(0310)^{*}-(019),-(0211)$ \\
\hline$(0311)^{*}-(0311)$ & 79.605 & -0.0022 & $(0311)^{*}-(0110),-(0212)$ \\
\hline$\left(\begin{array}{ll}31 & 0\end{array}\right)^{*}-\left(\begin{array}{ll}11 & 0\end{array}\right)$ & 85.602 & -0.0020 & $\left(\begin{array}{ll}11 & 0\end{array}\right)^{*}-\left(\begin{array}{ll}23 & 1\end{array}\right)$ \\
\hline$\left(\begin{array}{lll}11 & 3\end{array}\right){ }_{\star}^{\star}-\left(\begin{array}{ll}11 & 3\end{array}\right)$ & 91.383 & -0.0028 & $(113)^{*}-\left(\begin{array}{ll}12 & 2\end{array}\right),-\left(\begin{array}{ll}23 & 4\end{array}\right)$ \\
\hline$\left(\begin{array}{ll}11 & 4\end{array}\right)^{\star}-\left(\begin{array}{ll}11 & 4\end{array}\right)$ & 92.225 & -0.0018 & $(114) *-(123),-(135),-(235)$ \\
\hline$(115)^{*}-\left(\begin{array}{ll}11 & 5\end{array}\right)$ & 93.270 & -0.0037 & $(115)^{*}-(024),-(136)$ \\
\hline$\left(\begin{array}{ll}11 & 6\end{array}\right)^{*}-\left(\begin{array}{ll}11 & 6\end{array}\right)$ & 94.477 & -0.0023 & $(116)^{*}-(025),-(137)$ \\
\hline$\left(\begin{array}{ll}11 & 7\end{array}\right)^{\star}-\left(\begin{array}{ll}11 & 7\end{array}\right)$ & 94.941 & -0.0021 & $(117)^{*}-(026),-\left(\begin{array}{ll}12 & 6\end{array}\right),-(138)$ \\
\hline$(134)^{*}-(134)$ & 89.949 & -0.0019 & $\left(\begin{array}{ll}13 & 4\end{array}\right)^{*}-\left(\begin{array}{ll}12 & 5\end{array}\right),-\left(\begin{array}{ll}21 & 3\end{array}\right)$ \\
\hline$\left(\begin{array}{ll}13 & 5\end{array}\right)^{\star}-\left(\begin{array}{ll}13 & 5\end{array}\right)$ & 85.646 & -0.0017 & $(135)^{*}-\left(\begin{array}{ll}11 & 4\end{array}\right),-(226)$ \\
\hline$(136)^{*}-\left(\begin{array}{ll}136 & 6\end{array}\right)$ & 83.667 & -0.0016 & $(136)^{*}-(115),-(227)$ \\
\hline$\left(\begin{array}{ll}13 & 7\end{array}\right)^{*}-\left(\begin{array}{ll}13 & 7\end{array}\right)$ & 84.548 & -0.0023 & $\left(\begin{array}{ll}13 & 7\end{array}\right)^{*}-\left(\begin{array}{ll}11 & 6\end{array}\right),-\left(\begin{array}{ll}22 & 8\end{array}\right)$ \\
\hline$\left(\begin{array}{ll}13 & 8\end{array}\right)^{\star}-\left(\begin{array}{ll}13 & 8\end{array}\right)$ & 85.876 & -0.0017 & $\left(\begin{array}{ll}13 & 8\end{array}\right)^{*}-\left(\begin{array}{ll}11 & 7\end{array}\right),-\left(\begin{array}{l}229 \\
*\end{array}\right)$ \\
\hline$\left(\begin{array}{ll}13 & 9\end{array}\right)^{\star}-\left(\begin{array}{ll}13 & 9\end{array}\right)$ & 87.116 & -0.0018 & $(139){ }_{*}^{*}-(118),-(2210)$ \\
\hline$(1310) *(1310)$ & 88.698 & -0.0018 & $(1310)_{*}^{*}-(119),-(2211)$ \\
\hline$\left(\begin{array}{ll}21 & 3\end{array}\right)^{*}-\left(\begin{array}{ll}21 & 3\end{array}\right)$ & 93.143 & -0.0030 & $\left(\begin{array}{ll}21 & 3\end{array}\right)^{\star}-\left(\begin{array}{ll}13 & 4\end{array}\right),-\left(\begin{array}{ll}22 & 2\end{array}\right)$ \\
\hline$(225)^{*}-(225)$ & 76.596 & -0.0019 & $(225)^{\star}-\left(\begin{array}{ll}23 & 4\end{array}\right),-(316)$ \\
\hline$(234)^{*}-(234)$ & 91.455 & -0.0022 & $(234)^{*}-\left(\begin{array}{ll}11 & 3\end{array}\right),-\left(\begin{array}{ll}22 & 5\end{array}\right)$ \\
\hline
\end{tabular}




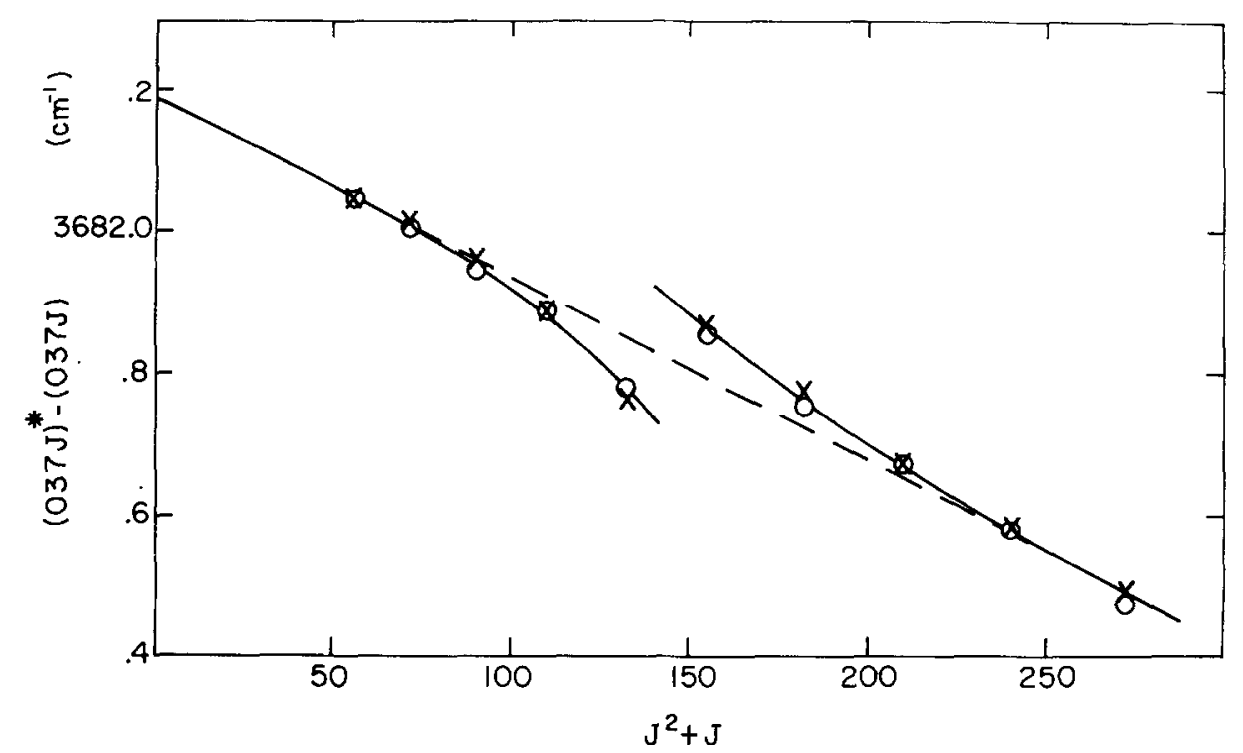

FIG. 5. Plots of $(037 J)^{*}-(037 J)$ versus $J^{2}+J$. The circles indicate points obtained from $\left[(037 J)^{*}\right.$ $-(016 J-1)]_{\text {obs }}+[(016 J-1)-(037 J)]_{\text {ealc }}$ whereas the crosses are from $\left[(037 J)^{*}-(028 J-1)\right]_{\text {obs }}$ $+[(028 J+1)-(037 J)]_{\text {ealc. }}$.

the plotted points fall, within experimental accuracy, on the same line. This indicates that the values for the normal state levels with $n=0$ must be self-consistent. However, in those cases where one or more series originated from a normal state level with $n>0$, it was frequently found that the plotted points fell on separate but parallel lines. Consider, for example, the three observed series, $(026 J)^{*} \leftarrow(035 J-1),(026 J)^{*} \leftarrow(017 J$ $+1)$, and $(026 J)^{*} \leftarrow(117 J+1)$. The points from the first two of these fall on the same straight line when $(026 J)^{*}-(026 J)$ is plotted against $J^{2}+J$. The equation of this line is $3681.400 \mathrm{~cm}^{-1}-0.0024\left(J^{2}+J\right)$. The third series however, yields a line with the same slope but a different intercept; the equation being $3681.538 \mathrm{~cm}^{-1}-0.0024$ $\times\left(J^{2}+J\right)$. The reason for this discrepancy appears to be the following. The value of (117) as calculated from the molecular constants of Ref. (3) was $491.875 \mathrm{~cm}^{-1}$. This value was used in reducing the line frequencies of the third series to obtain $(026 J)^{*}$ $-(026 J)$. Clearly the data from all three series can be made consistent if the actual value of (117) is taken to be $491.737 \mathrm{~cm}^{-1}$.

A second way of phrasing the situation is to note that since all three observed series are used to compute the same quantity, namely $(026 J)^{*}-(026 J)$, this implies the existence of combination relations between normal state levels. A review was made of all the series listed in Table II together with the normal state $Q$-branch origins as determined by Kwan and Dennison (3) with the object of finding what may be called observed values of energy levels of both the normal and the excited state. (Of course, spectral lines do not give energy levels themselves, but only the differences between energy levels, and consequently an intermediate step is necessary.) Lees and Baker (1) measured 14 microwave $Q$-branch origins which correspond to the differences between energy levels of the normal state with torsional quantum number $n=0$. In only two 
cases does the observed $Q$-branch origin differ by as much as $0.001 \mathrm{~cm}^{-1}$ from the calculated value. Since the limit of experimental accuracy attained in the present work is certainly not better than $0.001 \mathrm{~cm}^{-1}$, it seems reasonable to take the computed values

Table III. Observed rotation-torsion cncrgy lcvels, (ntK) of the normal state of methanol based on the levels for $n=0$. (See text.)

\begin{tabular}{|c|c|c|c|c|c|}
\hline$(010)$ & $=127.977 \mathrm{~cm}^{-1}$ & $(020)$ & $=137.099$ & $\left(\begin{array}{ll}03 & 0\end{array}\right)$ & $=137.099$ \\
\hline$(011)$ & $=131.853$ & $\left(\begin{array}{ll}02 & 1\end{array}\right)$ & $=142.604$ & $(031)$ & $=138.082$ \\
\hline$(012)$ & $=143.436$ & $\left(\begin{array}{ll}02 & 2\end{array}\right)$ & $=154.185$ & $(032)$ & $=145.977$ \\
\hline$(013)$ & $=162.589$ & $(023)$ & $=171.561$ & $(033)$ & $=161.138$ \\
\hline$(014)$ & $=189.074$ & $(024)$ & $=194.700$ & $(034)$ & $=183.820$ \\
\hline$\left(\begin{array}{ll}01 & 5\end{array}\right)$ & $=222.557$ & $(025)$ & $=223.824$ & $(035)$ & $=214.177$ \\
\hline$(016)$ & $=262.622$ & $(026)$ & $=259.322$ & $(036)$ & $=252.268$ \\
\hline$(027\}$ & $=308.846$ & $(027)$ & $=301.620$ & $(037)$ & $=298.061$ \\
\hline$(018)$ & $=360.908$ & $(028)$ & $=351.082$ & $(038)$ & $=351.436$ \\
\hline$(019)$ & $=418.706$ & $(029)$ & $=407.982$ & $(039)$ & $=412.174$ \\
\hline$(0110)$ & $=482.405$ & $(0210)$ & $=472.488$ & $(0310)$ & $=479.959$ \\
\hline$(0111)$ & $=552.352$ & $(0211)$ & $=544.673$ & $(0311)$ & $=554.394$ \\
\hline$(0112)$ & $=628.962$ & $(0212)$ & $=624.520$ & $(0312)$ & $=635.057$ \\
\hline$\left(\begin{array}{ll}11 & 3\end{array}\right)$ & $=403.29^{a}$ & $\left(\begin{array}{ll}12 & 0\end{array}\right)$ & $=336.92^{a}$ & $(130)$ & $=336.92^{\mathrm{a}}$ \\
\hline$\left(\begin{array}{ll}11 & 4\end{array}\right)$ & $=409.75^{a}$ & $\left(\begin{array}{ll}12 & 1\end{array}\right)$ & $=330.59^{\mathrm{a}}$ & $\left(\begin{array}{ll}13 & 1\end{array}\right)$ & $=354.05^{\mathrm{a}}$ \\
\hline$\left(\begin{array}{ll}11 & 5\end{array}\right)$ & $=426.221$ & $\left(\begin{array}{ll}12 & 2\end{array}\right)$ & $=335.57^{\mathrm{a}}$ & $\left(\begin{array}{ll}13 & 4\end{array}\right)$ & $=462.351$ \\
\hline$(116)$ & $=453.387$ & $\left(\begin{array}{ll}12 & 3\end{array}\right)$ & $=352.24^{\mathrm{a}}$ & $\left(\begin{array}{ll}13 & 5\end{array}\right)$ & $=507.59^{\mathrm{a}}$ \\
\hline \multirow[t]{3}{*}{$\left(\begin{array}{ll}11 & 7\end{array}\right)$} & $=491.737$ & $\left(\begin{array}{ll}12 & 4\end{array}\right)$ & $=380.57^{\mathrm{a}}$ & $(136)$ & $=539.640$ \\
\hline & & $\left(\begin{array}{ll}125 & 5\end{array}\right)$ & $=420.278$ & $(137)$ & $=565.740$ \\
\hline & & $(126)$ & $=470.923$ & $\{138)$ & $=597.398$ \\
\hline$\left(\begin{array}{ll}21 & 3\end{array}\right)$ & $=585.120$ & $\left(\begin{array}{ll}22 & 1\end{array}\right)$ & $=683.28^{\mathrm{a}}$ & $\left(\begin{array}{ll}23 & 4\end{array}\right)$ & $=555.09^{\mathrm{a}}$ \\
\hline$(219)$ & $=975.63^{\mathrm{a}}$ & $\left(\begin{array}{ll}22 & 2\end{array}\right)$ & $=737.29^{\mathrm{a}}$ & $\left(\begin{array}{ll}23 & 5\end{array}\right)$ & $=568.20^{\mathrm{a}}$ \\
\hline$(2110)$ & $=998.66^{\mathrm{a}}$ & $\left(\begin{array}{ll}22 & 3\end{array}\right)$ & $=763.92^{\mathrm{a}}$ & & \\
\hline \multirow[t]{6}{*}{ (2112) } & $=1072.46^{\mathrm{a}}$ & $\left(\begin{array}{ll}22 & 4\end{array}\right)$ & $=743.96^{\mathrm{a}}$ & & \\
\hline & & $\left(\begin{array}{ll}22 & 5\end{array}\right)$ & $=732.97^{\mathrm{a}}$ & & \\
\hline & & $\left(\begin{array}{ll}22 & 6\end{array}\right)$ & $=731.17^{\mathrm{a}}$ & & \\
\hline & & $\left(\begin{array}{ll}22 & 7\end{array}\right)$ & $=738.737$ & & \\
\hline & & $\left(\begin{array}{ll}22 & 8\end{array}\right)$ & $=756.173$ & & \\
\hline & & $\left(\begin{array}{ll}22 & 9\end{array}\right)$ & $=784.578$ & & \\
\hline$\left(\begin{array}{ll}3.1 & 1\end{array}\right)$ & $=1116.99^{\mathrm{a}}$ & $\left(\begin{array}{ll}32 & 0\end{array}\right)$ & $=879.04^{a}$ & $\left(\begin{array}{ll}33 & 0\end{array}\right)$ & $=879.04^{\mathrm{a}}$ \\
\hline$\left(\begin{array}{ll}31 & 2\end{array}\right)$ & $=1068.02^{a}$ & (32 I) & $=831.80^{\mathrm{a}}$ & $(331)$ & $=935.23^{a}$ \\
\hline$\left(\begin{array}{ll}31 & 3\end{array}\right)$ & $=1027.89^{\mathrm{a}}$ & $\left(\begin{array}{ll}32 & 2\end{array}\right)$ & $=793.66^{\mathrm{a}}$ & $\left(\begin{array}{ll}33 & 2\end{array}\right)$ & $=1000.33^{a}$ \\
\hline$\left(\begin{array}{ll}31 & 4\end{array}\right)$ & $=996.77^{a}$ & $\left(\begin{array}{ll}32 & 3\end{array}\right)$ & $=800.96^{\mathrm{a}}$ & $\left(\begin{array}{ll}33 & 3\end{array}\right)$ & $=1074.35^{\mathrm{a}}$ \\
\hline$\left(\begin{array}{ll}31 & 5\end{array}\right)$ & $=974.57^{\mathrm{a}}$ & $\left(\begin{array}{ll}32 & 4\end{array}\right)$ & $=872.91^{\mathrm{a}}$ & $\left(\begin{array}{ll}33 & 4\end{array}\right)$ & $=1157.18^{\mathrm{a}}$ \\
\hline$\left(\begin{array}{ll}31 & 6\end{array}\right)$ & $=961.36^{\mathrm{a}}$ & $\left(\begin{array}{ll}32 & 5\end{array}\right)$ & $=953.99^{\mathrm{a}}$ & $\left(\begin{array}{ll}33 & 6\end{array}\right)$ & $=1246.41^{\mathrm{a}}$ \\
\hline \multirow[t]{4}{*}{$\left(\begin{array}{ll}31 & 7\end{array}\right)$} & $=957.21^{\mathrm{a}}$ & & & $\left(\begin{array}{ll}33 & 7\end{array}\right)$ & $=1231.48^{a}$ \\
\hline & & & & $\left(\begin{array}{ll}338 & 8\end{array}\right)$ & $=1225.44^{\mathrm{a}}$ \\
\hline & & & & $(3310)$ & $=1240.29^{a}$ \\
\hline & & & & $(3311)$ & $=1261.14^{\mathrm{a}}$ \\
\hline
\end{tabular}

a Derived from the experimental work of Ref. (2). 
of $(0 \tau K)$ as the basis for determining all the remaining levels. The levels of the normal state as obtained in this way are given in Table III. The superscript $a$ means that the value in question was derived from the experimental work of Woods (2) as analyzed by Kwan and Dennison either wholly or in combination with the present measurements. Since the accuracy of Woods' work was about $0.03 \mathrm{~cm}^{-1}$, these values in Table III are given only to two decimals.

By employing the observed normal state levels listed in Table III, the intercepts of the plots of $(n \tau K)^{*}-(n \tau K)$ against $J^{2}+J$ are now all consistent for each group of observed series and these are the values appearing in Table II. By combining the entries from these two tables one obtains the 30 observed excited state levels which are given in the first columns of Table IV. As stated earlier, the largest contributor to $(n \tau K)^{*}$ is the fundamental vibration frequency $\nu_{0}$. The next terms in order of magnitude are the zeroth-order rotation terms including the internal rotation or torsion. These depend upon the new effective values of the barrier height $V_{3}$ and the moments of inertia $I a_{1}$ and $I a_{2}$. Changes in the perturbation terms as a result of exciting the vibration should be much smaller although of course they might have more importance than originally expected. With these considerations in mind, two least square fits to the observed data were made. In the first of these the varied quantities were $\nu_{0}, V_{3}, I a_{1}$, and $I a_{2}$ while the other constants $V_{6}$ through $k_{7}$ were given the values determined for the normal state levels in Ref. (3). The columns in Table IV labeled $\Delta_{1}$ give the observed values of $(n \tau K)^{*}$ minus the calculated values. The second least-squares fit was one where all the constants, 12 in number, were varied. $\Delta_{2}$ gives the observed minus the calculated quantities for this variation. The values of the constants as determined by these two least-squares fits are shown in Table $\mathrm{V}$, together with the molecular constants for the normal state.

Table IV. Observed and calculated energy levels for the excited oH vibrational state.

\begin{tabular}{|c|c|c|c|c|c|c|c|c|}
\hline$(\mathrm{n} \tau \mathrm{K})$ & $\star\left(\mathrm{cm}^{-1}\right)$ & $\begin{array}{c}\Delta_{1} \\
\text { (obs-calc) }\end{array}$ & $\begin{array}{c}\Delta_{2} \\
\text { (obs-calc) }\end{array}$ & & $(n \tau K)^{*}$ & $\left(\mathrm{~cm}^{-1}\right)$ & $\begin{array}{c}\Delta_{1}= \\
\text { (obs-calc) }\end{array}$ & $\begin{array}{c}\Delta_{2} \\
\text { (obs-calc) }\end{array}$ \\
\hline$(016)^{\star}$ & $=3943.821$ & -0.357 & -0.176 & (11 & $3)^{*}=$ & 4094.673 & +0.287 & -0.007 \\
\hline$(017)^{*}=$ & $=3989.136$ & -0.336 & -0.112 & (11 & $4)^{*}=$ & 4101.965 & -0.148 & -0.363 \\
\hline$(018)^{\star}$ & $=4040.831$ & +0.139 & +0.287 & (11) & $5)^{*}=$ & 4119.491 & -0.207 & -0.195 \\
\hline$(019)^{*}=$ & $=4098.245$ & +0.431 & +0.387 & (1) & 6) ${ }^{*}=$ & 4147.864 & -0.213 & +0.151 \\
\hline$(024)^{*}$ & $=3875.842$ & -0.428 & -0.828 & (1) & $7)^{*}=$ & 4186.678 & -0.767 & +0.015 \\
\hline$(025)^{*}=$ & $=3905.380$ & -0.012 & -0.262 & & & & & \\
\hline$(026)^{*}=$ & $=3940.722$ & -0.225 & -0.299 & $(13$ & 4) ${ }^{*}=$ & 4152.300 & -0.485 & -0.110 \\
\hline$(027)^{*}=$ & $=3982.584$ & +0.299 & +0.410 & $(13$ & $5)^{*}=$ & 4193.237 & +0.057 & +0.242 \\
\hline$(028)^{\star}=$ & $=4032.488$ & -0.230 & +0.038 & (13 & 6) ${ }^{*}=$ & 4223.307 & +0.273 & +0.138 \\
\hline$(0211)^{\star}=$ & $=4225.238$ & -0.162 & -0.280 & (13 & 7) ${ }^{*}=$ & 4250.288 & +0.069 & -0.179 \\
\hline \multirow[t]{2}{*}{$(034)^{\star}$} & $=2869.381$ & +1.579 & +1.239 & 113 & B) ${ }^{*}=$ & 4283.274 & +0.019 & -0.203 \\
\hline & & & & (13 & 9) ${ }^{\star}=$ & 4325.085 & +0.076 & -0.054 \\
\hline$(037)^{*}=$ & $=3980.251$ & -0.935 & -0.390 & (13) & $10)^{*}=$ & $4376.86 I$ & +0.239 & +0.196 \\
\hline$(0310)^{*}=$ & $=4160.063$ & -0.248 & -0.058 & $(21)$ & $3)^{*}=$ & 4278.263 & +0.448 & -0.177 \\
\hline$(0311)^{*}=$ & $=4233.999$ & +0.577 & +0.068 & $(22$ & 5) $)^{*}=$ & 4409.566 & -0.609 & -0.088 \\
\hline$(110)^{*}=$ & $=4108.203$ & +0.062 & +0.210 & $(23$ & 4) ${ }^{*}=$ & 4246.545 & +0.928 & +0.578 \\
\hline
\end{tabular}


Table V. Molecular constants of methanol in the normal and in the fundamental oH vibrational state.

\begin{tabular}{lccc}
\hline \multicolumn{1}{c}{ Parameters } & Normal State (Ref. 3) & $\begin{array}{c}\text { Excited State } \\
\text { 4 Parameter Fit }\end{array}$ & $\begin{array}{c}\text { Excited State } \\
\text { 12 Parameter Fit }\end{array}$ \\
\hline $\mathrm{Ia}_{1}\left(10^{-40} \mathrm{gm} \mathrm{cm} \mathrm{cm}^{2}\right)$ & 1.25022 & 1.30391 & 1.30429 \\
$\mathrm{Ia}_{2}\left(10^{-40} \mathrm{gm} \mathrm{cm}\right)$ & 5.33225 & 5.33129 & 5.39482 \\
$\mathrm{~V}_{3}\left(\mathrm{~cm}^{-1}\right)$ & 373.21 & 410.91 & 411.09 \\
$\mathrm{~V}_{6}\left(\mathrm{~cm}^{-1}\right)$ & -0.52 & -0.52 & -2.44 \\
$\mathrm{D}_{\mathrm{KK}}\left(10^{-4} \mathrm{~cm}^{-1}\right)$ & 0.38 & 0.38 & -2.31 \\
$\mathrm{k}_{1}\left(10^{-4} \mathrm{~cm}^{-1}\right)$ & -0.48 & -0.48 & -2.20 \\
$\mathrm{k}_{2}\left(10^{-4} \mathrm{~cm}^{-1}\right)$ & -18.41 & -18.41 & 27.28 \\
$\mathrm{k}_{3}\left(10^{-4} \mathrm{~cm}^{-1}\right)$ & -53.73 & -53.73 & -170.03 \\
$\mathrm{k}_{4}\left(10^{-4} \mathrm{~cm}^{-1}\right)$ & -85.50 & -85.50 & -70.88 \\
$\mathrm{k}_{5}\left(10^{-4} \mathrm{~cm}^{-1}\right)$ & 132.07 & 132.07 & -1.29 \\
$\mathrm{k}_{6}\left(10^{-4} \mathrm{~cm}^{-1}\right)$ & 67.85 & 67.85 & 440.06 \\
$\mathrm{k}_{7}\left(10^{-4} \mathrm{~cm}^{-1}\right)$ & 0 (fixcd) & $0(\mathrm{fixcd})$ & $0(\mathrm{fixed})$ \\
$v_{0}\left(\mathrm{~cm}^{-1}\right)$ & 0 & 3677.92 & 3679.62 \\
\hline
\end{tabular}

\section{DISCUSSION}

There is a striking contrast between the observed and calculated combination differences, $\delta$, which are given in Table $I$ and the observed and calculated differences $\Delta_{1}$ or $\Delta_{2}$ appearing in Table IV. The former are of the order of a few thousandths of a wave per centimeter and stem primarily from the experimental accuracy with which the spectral lines are measured. The $\Delta_{1}$ or $\Delta_{2}$, on the other hand, which are very much larger, represent the degree to which the present application of molecular theory is successful in calculating the rotation-torsion levels of the first excited $\mathrm{OH}$ vibrational state. It will be useful to discuss each of these separately.

In analyzing and identifying the observed lines in a spectrum which is as complex and crowded as the $\mathrm{OH}$ vibration of methanol it is very essential to have as many checks as possible. The combination relations which are exemplified in Table I constitute one such set of checks. The possibility of a quite independent type of check of the following sort was explored. As stated earlier, the ratio of the vibrational intensities of the perpendicular and parallel components of the band is estimated to be of the order of 6 to 1 . However, the squares of the dipole matrix amplitudes for corresponding $P$-, $Q-$, or $R$-branch lines are not given by the same functions of $J$ and $K$ and in general are about twice as large for the parallel as for the perpendicular transitions. This is related to the fact that in the parallel band $K \rightarrow K$ while in the perpendicular band there exist the two possibilities $K \rightarrow K+1$ or $K-1$. Taking this fact into account it is not unreasonable to look for the parallel band lines. Since these will occur in the central part of the $\mathrm{OH}$ vibration band where, as Fig. 2 shows, the lines are crowded together, it is probable that only the strongest series can be identified. This has proved to be the case. 
Consider, for example, the quantity $(016 J)^{*}-(016 J)$ which from Fig. 4 was found to have the value $3681.198-0.0016\left(J^{2}+J\right) \mathrm{cm}^{-1}$. The normal state energy levels are known with high accuracy and consequently $P_{-}, Q_{-}$, and $R$-branch lines, $(016 J)^{*} \leftarrow$ $\left(016 J^{\prime}\right)$, with $J^{\prime}=J+1,0$, and $J-1$ may be predicted. A search of the observed spectrum shows that more than 25 lines are found which lie within an average of 0.005 $\mathrm{cm}^{-1}$ from their predicted positions. A number of other series belonging to parallel transitions have also been identified and again their positions are consistent with the perpendicular transition series. In principle it would be very interesting to compare the intensities of corresponding parallel and perpendicular transition lines since this would determine the direction of the change of the clectric moment for the $\mathrm{OH}$ vibration. Unfortunately the lines in question occur in somewhat different parts of the spectrum and our experimental procedure did not measure these intensity ratios with any real accuracy. All that can be said at this time is that they are consistent with their expected values.

Because most of the identified perpendicular band $P$ - and $R$-branch series do not involve low values of $K$, the associated $Q$-branch lines are relatively weak. In general, however, for those series involving $K<7$ there is reasonable evidence in the spectrum for the existence of a corresponding $Q$ branch.

In summary, of the $67 P$ - and $R$-branch series measured in the $\mathrm{OH}$ vibration spectrum, it appears that in all cases sufficient checks are available to substantiate the identifications. The intercepts of the derived quantities $(n \tau K J)^{*}-(n \tau K J)$ have been determined with an accuracy which we estimate to be of the order of $0.005 \mathrm{~cm}^{-1}$ while the slopes are found to around $5 \%$ to $10 \%$ accuracy. The 67 measured series represent most but not all of the expected stronger series.

The situation regarding the calculation of the molecular constants and the comparisons of the observed and computed levels of the excited vibrational state is much less satisfactory and presents some puzzling features. Consider the slopes of the $(n \tau K J)^{*}-$ $(n \tau K J)$ plots which should be equal to the differences $\frac{1}{2}(B+C)^{*}-\frac{1}{2}(B+C)$ plus, perhaps, some smaller terms. As discussed earlier, an approximate calculation of this difference yielded the value $-0.0015 \mathrm{~cm}^{-1}$. The precise value resulting from the calculation is less important than the fact that the slope is expected to be essentially the same for all of the excited levels. A study of the slopes listed in Table II shows that this is certainly not the case. They vary from -0.0016 to -0.0050 and have an average value of $-0.0026 \mathrm{~cm}^{-1}$. The deviations from, for example, the average value appear to be quite irregular and do not show any marked trends as functions of the quantum numbers $n, \tau$, or $K$ with the possible exception of the levels $(03 K)^{*}$ which are involved in the largest slopes.

In addition to the expected main contributor to the slope, namely, $\frac{1}{2}(B+C)^{*}-$ $\frac{1}{2}(B+C)$, the smaller terms which could possibly be important are the Kirtman terms $\left[G_{V}\left\langle P \gamma^{2}\right\rangle+L_{V} K\langle P \gamma\rangle+F_{V}\langle(1-\cos 3 \gamma)\rangle\right]\left(J^{2}+J\right)$, the terms arising from the asymmetry of the molecule and finally the centrifugal distortion term $-D_{J K} K^{2}\left(J^{2}+J\right)$. The reason for ignoring them up until now is that their magnitude for the normal state molecule is very small in comparison with the principal term $\frac{1}{2}(B+C)\left(J^{2}+J\right)$ and thus small changes in the perturbation constants would not lead to significant. effects. In spite of this fact an attempt was made to fit the slopes using empirically determined constants $G_{V}{ }^{*}, \ldots, D_{J K}{ }^{*}$. The agreement with the observed slopes was so poor as to indicate that this could not be the solution to the puzzle. 
Consider next our determination of the effective molecular constants for the excited state through the use of least-squares fits to the observed energy levels. As noted, for the first fit the dominant quantities $\nu_{0}, V_{3}, I a_{1}$, and $I a_{2}$ were varied, while for the second fit the constants of the perturbation terms were also varied. Neither set of differences, $\Delta_{1}$ or $\Delta_{2}$, between the observed and calculated levels can be considered really satisfactory although either one is a great improvement over the situation where all normal state constants are used to calculate the excited state levels. In that case the differences would range from +9 to $-9 \mathrm{~cm}^{-1}$. For the set $\Delta_{1}$, the rms deviation is $0.51 \mathrm{~cm}^{-1}$ while the corresponding figure for $\Delta_{2}$ is $0.45 \mathrm{~cm}^{-1}$. The $\Delta_{1}$ and $\Delta_{2}$ tend to show irregularities within a succession of levels having the same $n$ and $\tau$ but different $K$ which would make it virtually impossible to fit the observed levels with the same type of formula which was successful for the normal state levels. In any case the $\Delta$ 's are large compared with the experimental accuracy. In particular both $\Delta_{1}$ and $\Delta_{2}$ are very large for the level $(034)^{*}$ and yet this is one of the best-determined levels since $P_{-}, Q_{-}$, and $R$-branch series have been identified for both perpendicular and parallel transitions. Incidentally the slope of the plot $(034 J)^{*}-(034 J)$ has the abnormally large value of -0.0050 . However, there seems to be no very clear correlation between abnormal slope and large $\Delta$.

Turning to a consideration of the molecular constants listed in Table $\mathrm{V}$, it is seen that the values of the important constants $\nu_{0}, V_{3}, I a_{1}$, and $I a_{2}$ are in reasonable agreement for both least-squares fits. $\nu_{0}$ must certainly lie close to $3679 \mathrm{~cm}^{-1}$. The effective barrier height for the $\mathrm{OH}$ fundamental vibration is raised by about $10 \%$, or $38 \mathrm{~cm}^{-1}$. (The respective figures for the two fits are 37.7 and $37.9 \mathrm{~cm}^{-1}$.) The effective value of the hydroxyl moment of inertia, $I a_{1}$, is raised for both fits by $4 \%$ in fair agreement with the estimate of $6 \%$ described earlier. The $I a_{2}$ increases are small, $0.02 \%$ and $1 \%$, again as was expected.

The perturbation constants obtained from the second least-squares fit appear to be very different from the corresponding normal state constants and it is our opinion that the values as listed in the last column of Table $\mathrm{V}$ have little or no validity. This is in contrast to our determinations of $\nu_{0}, V_{3}, I a_{1}$, and $I a_{2}$ which seem to be reasonably well substantiated by the observed spectrum.

At this point one can only speculate as to the origin of the unsatisfactory values of $\Delta_{1}$ or of $\Delta_{2}$. It seems clear that the theoretical formula which is used to correlate the observed levels of the excited state must be incomplete. The most probable explanation is that overtones or combinations of low-lying vibrational states are introducing resonances with the $\mathrm{OH}$ fundamental and that an adequate theoretical calculation must also take these into account. It may be remarked that methanol has three fundamentals with frequencies between 1033 and $1160 \mathrm{~cm}^{-1}$, four fundamentals between 1345 and $1475 \mathrm{~cm}^{-1}$, and two fundamentals between 2800 and $3000 \mathrm{~cm}^{-1}$. The extreme density of strong lines observed between 3840 and $3920 \mathrm{~cm}^{-1}$ most probably is also the result of overtones or combinations of these frequencies.

\section{ACKNOWLEDGMENT}

We thank Mr. David Snyder for valuable assistance with the final stages of the data fitting.

RECEIVED : February 2, 1975 


\section{REFERENCES}

1. R. M. Lees and J. G. Baker, J. Chem. Pliys. 48, 5299 (1968).

2. D. R. Woods, Ph.D. dissertation, The University of Michigan, 1970.

3. Y. Y. KWan and D. M. Dennison, J. Mol. Spectrosc. 43, 291 (1972).

4. R. M. Lefs, I. Chem. Phys. 57, 2249 (1972).

5. R. M. LeEs, J. Chem. Phys. 57, 824 (1972).

6. B. KirTMan, J. Chem. Phys. 37, 2516 (1962).

7. D. B. Burkhard and D. M. Dennison, J. Mol. Spectrosc. 3, 299 (1959).

8. R. H. Hunt, C. W. Robertson, and E. K. Plyler, Appl. Opt. 6, 1295 (1967).

9. P. A. Jansson, R. H. Hunt, and E. K. Plyler, J. Opt. Soc. Am. 60, 596 (1970).

10. H. R. Gordon and T. K. McCubBin, J. Mol. Spectrosc. 19, 137 (1966).

11. A. Giacchetti, R. W. Stanley, and R. Zalubus, J. Opt. Soc. Am. 60, 474 (1970)

12. R. L. SAMS, private communication. 